\title{
The Cognitive Processes of Probability Estimation in Random Sampling from Discrete Uniform Spaces
}

\author{
Jacob M. Schreiber \\ Department of Education, Ben-Gurion University of the Negev \\ 5 Sharet Street, Ramat - Gan, Israel \\ Tel: 972-3-673-4060_E-mail: ora1963@gmail.com
}

Received: January 26, 2012

Accepted: February 16, 2012 Published: May 1, 2012

doi:10.5539/jedp.v2n1p21

\author{
URL: http://dx.doi.org/10.5539/jedp.v2n1p21
}

\begin{abstract}
The research examines how high school students solve simple probability problems, and the probability estimations of students, boys and girls, in a discrete and uniform probability space. In discrete uniform probability spaces problem solving using estimations of probabilities and strategies of solving problems in a variety of situations were described. Solving probability problems among elementary intermediate and high school students were studied, however, reviewing the literature in probability; I am not familiar with any research on estimating probabilities or characterizations of rules people use for estimating and solving probability problems relating specifically to numerically symmetric and asymmetric objects. The research describes the rules and heuristics that student use to solve probability problems and estimate probabilities. The research has shown that students at different levels of proficiency in solving probability problems take a different approach when solving and estimating probabilities. When students are asked to estimate the probability of selecting two beads with different colors from a bag containing beads of different colors some calculate probabilities and other estimate them using a variety of rules. The use of rules for estimating probabilities is directly related to numerical relations and symmetries of objects. I describe a variety of rules students use for estimating probabilities, e.g., the "pair rule: if the number of pairs of objects in a group is greater than the number of the other pairs the probability of drawing a pair of first kind is greater than drawing pairs of the second kind". I present a mechanism for the estimations and decisions taken by students when estimating probabilities.
\end{abstract}

Keywords: Asymmetry, Cognition, Mechanism, Perception, Probability, Sampling, Symmetries, Unified, Uniform

\section{Introduction}

When students solve problems which have a probabilistic characterization, many of them "put aside" formal rules and use instead heuristics to solve these problems.

Preview research gives strength to these views. In their work (Piaget \& Inhelder, 1975; Schnarch, 1998), which investigated also students that studied probability, found that when these students solved probability problems which included numerically symmetric and asymmetric sets of objects many of them have used rules for solving problems. An interesting strengthening in the use of heuristics in solving probability problems is shown by an experiment conducted by Tversky and Kahneman (1969). They found that many senior academic people from areas such as mathematical psychology who were asked to solve problems presented to them in a questionnaire based their judgment on small samples, showed insensitivity to the size of the sample, and used a heuristics approach to solve these problems, which made it impossible for them to solve it correctly (Kahneman \& Frederick, 2002; Tversky \& Kahneman, 1971).

Gestalt psychologists, who have studied the visual system, have shown that visual perception is not a direct reading of the world. For example a dense set of points ...is conceived as a continuous line compared to a separated set of points... There is a tension between observing material and our perception (Goldmeier, 1972; Wertheimer, 1950). In this case the point represents the material and the line is the figure. Minimizing distances between the points improves the chances that we see a line or a figure and not just a collection of points 
(material). A similar behavior of the brain is described by the mar-poggio model for stereograms; when looking at a complex collection of points the brain produces a picture (Pinker, 1999; Poggio, 1984).

In a preview research (Schreiber, 2003) a direct relation between the representation of the problem and the use of intuitive rules is shown with respect to the set of objects being drawn. More than that, a relation between false representations of the problem and other intuitive rules was found. The less the representation of the sample is based on rules of probability theory and the more the student relies on his beliefs with relation to the sample, the greater the tendency to build false intuitive models and use them to solve the problem. It is specifically related to the first research assumption which was: a) The more the numerical distance between the component objects in the sample space decreases, the greater the student's difficulty to choose the correct bag when asked to estimate the probability of selecting two beads with different colors from several bags. b) As the numerical distance among the components of objects in a set of objects decreases, there is an increased difficulty to estimate probabilities correctly. As the numerical distance increases there is also an increased difficulty to estimate probabilities correctly.

In the literature the phenomena of over-sensitivity of people to certain visually symmetric objects have been described (Leyton, 1992). In the present research a more specific question came up: what is the effect of sensitivity to symmetry on solving mathematics problem and more specifically what is the effect on probability problem solving? This question is a continuation of the investigation I had started in my research (Schreiber, 2003). There I had found differences in the problem solving approach students were using when solving questions with numerically symmetric object arrangement; the rate of correct answers was smaller compared with those with numerically asymmetric object arrangement. In my preview work I had found that high school students in grades 9-12 who studied probability and those who did not explained their answers using the numerical distances in the sets of objects, symmetric and asymmetric structures in the problems; most students did not calculate probabilities formally. In solving certain probability problems with symmetric object arrangements, two thirds of students did not calculate probabilities.

In my previews students answered questions using the intuitive rule "More of A, More of B" - more beads of a certain color, greater the chances of drawing two beads with this color from the set of object. They used the rule "same A, same B" - same number of objects with different colors in the bag, greater the probability of randomly drawing a pair of beads with different colors from that bag. These findings gave rise to the question: how will students who studied for their final school mathematics test in probability react to questions in which there are numerically symmetric and asymmetric sets of objects? Will they use formal probability or certain heuristics to answer these questions? Will they solve probability problems formally or heuristically when the numerical distances among the sets of objects increase? What will they do when the number of objects is numerically equal? Will students be more likely to use an intuitive problem solving approach, and will it increase or decrease for different object arrangements? These were interesting questions for me and I thought it would be possible to find out what rules students use for symmetric and asymmetric object arrangements when solving probability problems. The previews research was relatively small and I did not check a variety of symmetric and asymmetric object relations. This led to the present research. The previews research raised several questions concerning probability problem solving and I chose the following question for the present research: what are the important cognitive factors in solving probability problems such as those described above?

\subsection{The Effect of Numerical Symmetry among Objects on Probability Problem Solving}

In my experience Schreiber (2003), when we present to students a probability problem in which they are requested to calculate the probability of drawing a pair of beads from bags which contain numerically symmetric sets of objects, or almost numerically symmetric, the thinking in certain cases is directed towards the symmetric representation of the set of objects. Example, one bag contains four red beads and three green beads, a second bag contains four red beads and four green beads, a third bag contains three red beads and four green beads, and a person is asked to select a bag in order to draw a pair of beads of different colors with highest probability. Most students select the second bag which has a numerically symmetric arrangement of beds. The finding was that if students do not use formal probability calculations they use instead a set of rules for estimating the probabilities.

The main diversions that I described in solving probability problems Schreiber (2003) are: the use of symmetric and asymmetric sets in the problem structure and the tendency of students to see a symmetric and asymmetric representation are related to the selection of action and the rules for solving the probability problems. Students who used rules for solving the problems described the numerical distances among the objects and how they arrived at a solution to the problem and estimated the probabilities. The students' use of estimation rules was a hint that the structure of the set of objects and the numerical relations among them contain the important 
elements that influence the way problems of this type are being solved. Students explained their answers using the number of objects, and the numerical relations of the objects such as: "there are more reds", "the number of red and green is equal", using extensive quantities and informal probability estimations (Piaget, 1964).

\section{Methodology}

The population in this research is composed of high school students, grades 10-12, studying probability for the national mathematics examination, boys and girls. In the early test 411 students participated, of whom 46 took part in the interviews.

Searching the literature on problem solving in probability I could not find an explanation or a general description of the factors involved in solving probability problems in a discrete uniform probability space; I could not find an explanation for using estimation rules when solving probability problems in sets of objects which have numerical symmetry and asymmetry. Therefore using the estimation rules for solving problems, the heuristics and intuitions, and research and findings in an earlier study (Schreiber, 2003), I developed the following questions:

\subsection{The Research Questions}

What are the characteristic cognitive processes of probability problem solving and estimations when sampling from a discrete and uniform probability sample space?

Do boys and girls solving probability problems in a discrete uniform probability space use estimation rules at an equal rate?

In this research I plan to: a) describe qualitatively the way problems with numerically symmetric and asymmetric objects are being solved in a discrete uniform probability space; b) check the consistency of students when solving problems; c) check the empirical relation between the use of rules and correctness when solving probability problems; d) check if there are differences between boys and girls in solving probability problems associated with numerically asymmetric and symmetric sets of objects.

From the research questions and the literature I reviewed I have conjectured several assumptions with respect to the characteristics of probability problem solving. These assumptions are in the context of a unified and uniform probability space that includes several types of objects with different colors in different quantities, or same quantities, and from which we sample a pair of objects.

\subsection{Main Research Assumptions}

1) (Asymmetry) When solving probability problems in which there are numerically more objects of one type than the other, students will solve the problem using rules for estimating probabilities estimating that two beds of the same color will be drawn and use rule number one (or variations of it to be described).

2) (Symmetry) When solving probability problems in which numerically there are the same number of beads of different colors, students will solve the problem using rules for estimating probabilities from equal sets of objects, estimating that two beads, one of each color, will be drawn at random, and use rule number two (or variations of it to be described).

3) Students show consistency in solving probability problems from numerically asymmetric sets of objects: Students who use estimation rules when solving problems from asymmetric sets of objects will do so when solving problems from symmetric sets of objects; students who use estimation rules when solving problems from numerically symmetric sets of objects will do so when solving problems from numerically asymmetric sets of objects.

4) The more the use of the estimation rules increases, the more the rate of correct answers decreases.

5) Boys and girls solve probability problems using the estimation rules at the same rate.

\subsection{The Independent Variables}

Student characteristics: Grades 9-12; probability education: studied probability, did not study probability. Grades in the classes that were tested: high grades, medium grades, low grades.

Gender: Boys, Girls.

Object sets. The number of objects and numerical relations among the types of objects being pulled out, and the number of sets in the population. Group A- contains questions relating to asymmetric sets of objects (henceforth the asymmetry set), Group B- contains questions relating to symmetric sets of objects (henceforth the symmetry set). 
I have defined in the set of objects three types of distances: high, medium, and small distance; and type of symmetry (higher and lower): a) Asymmetry. Numerically high distance is a distance greater than three beads. A medium distance is a distance of two or three beads. A small distance is a distance of one between the numbers of beads.

b) Symmetry. The symmetry is largest when the numerical difference is zero and less when the numerical distance is one or two.

\subsection{The Dependent Variables}

The rules, strategies and consistency of using rules and strategies when solving probability problems.

\subsection{Research Design}

The design was built to verify the research questions with respect to the different sets of objects - numerically symmetric and asymmetric sets of objects. For that purpose the structure of the data in the questions (such as numerical relations of objects) was manipulated systematically in order to reveal students strategies for solving probability problems and the use of heuristics and rules in many different object arrangements.

\subsubsection{Research Tools}

There were two items: questionnaires and a personal interview:

\section{a. The Questionnaires}

\section{i. Early Questionnaires (early written test)}

The early questionnaires contained two parts and seven sub-questions. The students were asked to answer in writing.

The questions in the early written test are similar in style to those in the interview questionnaire, presented here.

\section{ii. Research questionnaire}

The research (interview) was composed of seven questions and sub-questions, thirteen questions in all. The students were asked to reply to most of the questions verbally. It contained questions with numerically asymmetric and numerically symmetric objects. The answers were recorded.

\section{b. The Interview Questions}

The following questions are about the random drawing of beads from bags without seeing the content of the bag. The questions are about the colors of beads drawn at random, or about choosing a bag from which the probability of choosing a pair of beads one red and one green at random is with higher probability.

Questions:

1. A bag contains 9 red, 6 green, 5 yellow, and 1 white bead. We want to draw a pair of beads at random. With respect to the following situations you are asked to compare the probabilities and mark the sentence as correct or incorrect.

a) The probability that on a random drawing from the bag two red beads will be drawn is higher than drawing one red bead and one green bead. True, False.

b) The probability that in a random drawing from the bag two beads one red and one yellow are drawn is larger than the probability that two red beads will be drawn. True, False.

c) The probability that in a random drawing from the bag two beads one red and one yellow is greater than the probability of drawing two green beads. True, False.

d) Joe has sampled at random from bag contains 9 red, 6 green, 5 yellow, and 1 white bead and pulled two beads one red and one green and you are interested in drawing two more beads together without returning the beads that had been taken out. i) The probability that in a random drawing from the bag two red beads will come out is greater than the probability that two beads one red and one yellow will come out. True: False. ii) the probability that in another random drawing from the bag one red and one yellow beads is greater than the probability that that two beads one red and one green will come out. True, False.

e) Joe has sampled again at random from the bag and has taken out two beads one red and one green and you are interested in taking out another two beads together, without returning the beads taken out. i) The probability that in another random drawing from the bag two red beads are drawn is greater than the probability of drawing two beads one red and one green. True, False. ii) The probability that in another random drawing from the bag two red beads are drawn is greater than the probability of drawing two beads one red and one yellow. True: False. iii) 
The probability that in another random drawing from the bag two beads one red and one yellow are drawn is greater than the probability of drawing two beads one red and one green. True, False.

f) Joe has sampled again at random from the bag and has taken out two beads one red and one yellow and you are interested in taking out another two beads together, without returning those taken out. i) The probability that in another random drawing from the bag two red beads are drawn is greater than the probability of drawing two beads one red and one green. True, False. ii) The probability that in another random drawing from the bag two red beads are drawn is greater than the probability of drawing two beads one red and one yellow. True: False. iii) The probability that in another random drawing from the bag two beads one red and one yellow are drawn is greater than the probability of drawing two beads one red and one green. True, False.

g) Joe has sampled again at random from the bag and has taken out two beads one red and one yellow and you are interested in taking out another two beads together, without returning the beads taken out. i) The probability that in another random drawing from the bag two red beads are drawn is greater than the probability of drawing two beads one red and one green. True, False. ii) The probability that in another random drawing from the bag two red beads are drawn is greater than the probability of drawing two beads one red and one yellow. True: False.

2. a. The bag contains 9-red beads, 3-green and one yellow. We want to randomly draw a pair of beads. For each situation described you are requested to say if the sentence is correct or not.

i) The probability that in a random drawing from the bag two red beads are drawn is greater than the probability of drawing two beads one red and one green. True: False.

ii) The probability that in a random drawing from the bag two green beads are drawn is greater than the probability of drawing two beads one yellow and one green. True: False.

b. The bag contains 7-red beads, 3-green and one yellow. We want to randomly draw a pair of beads. For the situation described below you are requested to say if the sentence is correct or not.

The probability that in a random drawing from the bag two red beads are drawn is greater than the probability of drawing two beads one red and one green. True: False.

3. Three bags contain beads: the first contains 4-red beads and three green beads, the second contains 4-red and 4-green beads, and the third contains 3-red beads and 4-green beads.

From which bag the probability of randomly choosing two beads one red and one green is the largest?

4. Three bags contain beads: the first contains 400-red beads and 400- green beads, the second contains 4-red and 3 -green beads, and the third contains 300 -red beads and 300 -green beads.

- From which bag the probability of randomly choosing two beads one red and one green is the largest?

5. Three bags contain bead: the first contains 100 -red beads and 100- green beads, the second contains 4-red and 3 -green beads, and the third contains 3 -red beads and 4-green beads.

- From which bag the probability of randomly choosing two beads one red and one green is the largest?

6. Three bags contain beads: the first contains 99-red beads and 100- green beads, the second contains 4-red and 2 -green beads, and the third contains 100 -red beads and 99 -green beads.

- From which bag the probability of randomly choosing two beads one red and one green is the largest?

7. Two bags contain beads: the first contains 7-red beads and 5-green beads, the second contains 9-red and 8 -green beads.

- From which bag the probability of randomly choosing two beads one red and one green is the largest?

Key answers for the interview questions:

1a-Not True

1b-True

1c-True

1di-Not True

1dii-Not True

1ei- Not True

1eii- Not True 
1eiii-True

1 fi- Not True

1fii- Not True

2ai-True

2aii-Not true

2b-Not true

3-All probabilities are equal

4-Second bag

5-Second and third bag

6-Second bag

7-First bag

Examples of probability calculations in questions with numerical asymmetry of the objects, e.g., questions $\mathbf{1}$ a-c. In question 1a the answer is False, in the first item the highest probability is to draw two beads one red and one green. The calculations for drawing the other pairs in question one are shown below:

$$
\begin{aligned}
& P(\text { red }, \text { green })=\frac{9}{21} \times \frac{6}{20}+\frac{6}{21} \times \frac{9}{20}=0.257 \\
& P(\text { red }, \text { yellow })=\frac{9}{21} \times \frac{5}{20}+\frac{5}{21} \times \frac{9}{20}=0.214 \\
& P(\text { red }, \text { red })=\frac{9}{21} \times \frac{8}{20}=0.171 \\
& P(\text { green, yellow })=\frac{6}{21} \times \frac{5}{20}+\frac{5}{21} \times \frac{6}{20}=0.142 \\
& P(\text { green }, \text { green })=\frac{6}{21} \times \frac{5}{20}=0.071 \\
& P(\text { yelloww, yellow })=\frac{5}{21} \times \frac{4}{20}=0.047 \\
& P(\text { red, white })=\frac{9}{21} \times \frac{1}{20}+\frac{1}{21} \times \frac{9}{20}=0.042 \\
& P(\text { green }, \text { white })=\frac{6}{21} \times \frac{1}{20}+\frac{1}{21} \times \frac{6}{20}=0.028 \\
& P(\text { yellow, white })=\frac{5}{21} \times \frac{1}{20}+\frac{1}{21} \times \frac{5}{20}=0.023
\end{aligned}
$$


Generally the probability relations are:

$\mathrm{P}($ red, green $)>\mathrm{P}($ red, yellow $)>\mathrm{P}($ red, red $)>\mathrm{P}($ green, yellow $)>\mathrm{P}($ green, green $)>\ldots$

In question $2 b$ the Answer is - Not True

The calculations are given by

$$
\begin{gathered}
P(\text { red }, \text { green })=\frac{7}{11} \times \frac{3}{10}+\frac{3}{11} \times \frac{7}{10}=0.381 \\
P(\text { red }, \text { red })=\frac{7}{11} \times \frac{6}{10}=0.381
\end{gathered}
$$

Question 3: In this question the setting is one of numerical symmetry of objects and it is given here as an example for calculating the probabilities in questions 3-7. The answer in question 3 is that all the probabilities are identical.

The probability of drawing one red and one green bead from the first bag is:

$$
P(\text { red }, \text { green })=\frac{3}{7} \times \frac{4}{6}+\frac{4}{7} \times \frac{3}{6}=\frac{4}{7}
$$

The probability of drawing one red and one green bead from the second bag is:

$$
P(\text { red }, \text { green })=\frac{4}{8} \times \frac{4}{7}+\frac{4}{8} \times \frac{4}{7}=\frac{4}{7}
$$

The probability of drawing one red and one green bead from the third bag is:

\subsubsection{Data Analysis}

$$
P(\text { red }, \text { green })=\frac{4}{7} \times \frac{3}{6}+\frac{3}{7} \times \frac{4}{6}=\frac{4}{7}
$$

Checking the assumptions and the rate students are using the rules.

The fitting of students' answers to the research assumptions is based on student's answers in the written test and the interviews. The rate of using correct answers is based on the number of correct answers of each interviewee, not taking into account the type of answers, calculation, and use of estimation rules or any other way.

\section{Findings}

The findings are characterized by the informal rules that were used by students with intermediate and low grades in the written test to estimate probabilities: rules used for estimating probabilities from numerically symmetric and asymmetric sets of objects.

\subsection{The Written Test}

The main points that came up from analyzing the answers in the written test are: answers to problems with numerical asymmetry of the objects had a rate of correct answers that was less than one third and answers to problems that had symmetric sets of objects were correct at a rate of almost one half. Students who participated in the written test were classified into three groups: high grades: 5-6 correct answers, less than one third of students; intermediate 3-4 correct answers, approximately one third of students; low grades 0-2 correct answers, almost two third of the students.

\subsection{The Interviews}

The cognitive processes that students used were manifested mainly in the use of rules for estimating probabilities. More precisely, it could be observed that students used heuristics and intuitive rules that included the examination of the rule with respect to the information in the problem. See tables 1-2.

1. Students with high grades in the interviews use formal estimation rules consistently. In problems containing numerically symmetric sets they used calculations at a rate of two thirds.

2. Students with intermediate and low grades who solved probability problems with numerically symmetric sets 
of objects used probability estimation rules consistently.

3. The rate students used rules for estimating probabilities from asymmetric sets increases as the numerical difference between objects gets larger.

4. The rate of using rules for numerical symmetry among objects is almost double the rate of using rules for numerical asymmetry among the objects.

5. When solving probability problems no statistical differences in the rate of using rules and the rate of correct solutions for boys and girls was found. However, when solving certain types of problems that have numerical symmetry of objects boys and girls estimated probabilities using rules at a different rate (e.g., problem 6 , an interaction was found using Log-linear analysis $\mathrm{p}<0.1, \mathrm{G}_{(7)}{ }^{2}=11.42$ ). This finding requires further analysis in solving certain types of problems with numerical symmetry of objects.

\subsection{Problem Solving in the Interviews}

Students with high grades. In all the questions with numerical asymmetry of objects, the students answered by calculating probabilities. In questions with numerical symmetry of objects, a fifth of the students answered by using one of the rules.

Students with intermediate grades. In answering questions with numerical symmetry of objects, more than half the students used rules. In answering questions with numerical asymmetry of objects, about one third of the students used the rules.

Students with low grades. In answering questions with numerical symmetry of objects, more than two thirds of the students used rules. In answering questions with numerical asymmetry of the objects, less than one half the students used estimation rules.

\subsection{Rules for Estimating Probabilities}

This section contains several of the rules mentioned in the research and used by students in the interviews, the written test, and overviews of the rules.

In the literature intuitive rules are characterized as "intuitive" or "heuristics". Here each rule is characterized by its original name. The rules presented are related to probability estimation in a unified and uniform space.

\section{A. Asymmetry- Numerical difference among the objects}

This rule described in a previews research (Schreiber, 2003) is set into action when it is known that there are more objects of one kind compared with the other.

The rule is: "When there is a group of objects $(A, B, C, D \ldots)$ in a unified probability field and a pair is being drawn at random if A has the largest frequency the probability of drawing a pair AA is the largest".

This rule has several variations according to the numerical differences among the objects and their relations: Numerical asymmetry among the dominant objects and asymmetry among sub-components (asymmetry of the second kind).

\section{B. Symmetry- numerical equality of the objects being sampled}

The rule is activated when there is the same number of objects of two types being considered. The rule is: "When in a group of objects there are numerically the same number of objects type A and B and two are drawn at random the probability is greater that one of each type will be drawn, a pair $\mathrm{AB}$ ". This rule has variations when there are several types of object arrangements.

\section{Compounded heuristics rules for probability estimation}

In a recurrent drawing process of random drawing without replacement from a set of objects there is a use of a compound rule for estimating probabilities and problem solving (Schreiber, 2003). For example: using rule A and $\mathrm{B}$ together when drawing pairs of objects at random from a set (A, B, C...) that contains numerically more objects of type "a" and "b" $(a \geq b \geq c \ldots)$ there is a greater probability that two objects of type AA or AB will be drawn, and after drawing that pair if there is an equal number of objects type $\mathrm{A}$ and $\mathrm{B}$ the probability is that on drawing two more objects at random the two will be $\mathrm{AB}$, and if after that drawing the numerical relation changes such that $(\mathrm{a} \geq \mathrm{c} \geq \mathrm{b} \ldots)$ the probability is that on drawing another two at random AA or AC will be drawn ...etc'. These compounded rules have many variations.

\section{Rules for estimating probabilities from numerically symmetric and asymmetric object arrangements}

A. Rules for estimating probabilities from sets of objects containing numerically asymmetric sets of objects 
1. Numerical difference rule- Asymmetry: "If there are more objects of one type the probability of drawing two beads of that type on a random drawing of pairs is the greatest."

1a. Numerical difference rule - Asymmetry of the second type: "If there are more objects of one type (not necessarily numerically the largest type). The probability of drawing a pair of objects of that type is larger than the probability of drawing a pair of the other objects.

1b. Summation rules: "If the sum of two types of objects is greater than any other type or any combination of other objects, the probability of drawing a pair of this type is larger compared to the probability of drawing pairs of objects from the other objects.

1c. The pair rule: if the number of pairs of objects in a group is greater than the number of the other pairs the probability of drawing a pair of that kind is greater than drawing pairs of the other kind.

There is a variation of the "pair rule" suitable for numerically symmetric sets of objects. "If the number of pairs of two types of objects is equal, the probability of drawing a pair of objects, one object of each type, is equal."

B. Rules for estimating probabilities when drawing pairs of objects from approximately numerically symmetric sets of objects

2. Numerical Symmetry: If the bag contains the same number of objects of different types $(a=b \geq c \ldots)$, the probability of drawing two items in different color is larger than the probability of drawing a pair of objects from the other bags.

2a. Numerical Symmetry of the Second Order: If a bag contains the same number of objects of different types (a $\geq \mathrm{b}=\mathrm{c} \ldots$ ) the probability of drawing two items with different colors is larger than the probability of drawing a pair of objects from the other bags, given that in the other bags the number of objects is large (more than 50 or 100 in a bag), and even if there is a numerical equality of objects in the other bags.

The main characteristic of students estimating probabilities in this group is that they think there is a much higher probability of drawing a pair of beads one in each color from numerically symmetric sets of objects compared to numerically larger asymmetric sets of objects.

2b. There are two different types of objects with equal number of beads and numerically they are the highest number in a set of beads:

If the bag contains the same number of beads of different types and the arrangement of objects is $(a=b \geq c \ldots)$, the probability of drawing two beads with different colors is larger than the probability of drawing a pair of beads from the other bags, given that they contain less than 50 beads in a bag (this is consistent with the structure of the questions in the present research, the answers students gave, and not presented as a general rule used by students).

The main characteristic of students estimating probabilities in this group is that when they use the rules they think that there is a much higher probability of drawing a pair of objects one in each color from the larger set of objects compared to groups with numerically smaller number of objects.

Insert Table 1 Here

Insert Table 2 Here

\section{Discussion}

According to Evans (2003) people have two separated cognitive systems. One is old in evolutionary terms (system 1) and is also prevalent among animals; it includes a set of autonomic sub-systems that includes inborn traits and domain-specific knowledge. The other is human in nature and characterizes human thinking (system 2). The characteristic of students with intermediate and low grades is the use of system 1 rules for solving probability problems and the use of extensive and intensive quantities described by Piaget (1964). He described two quantities: intensive quantities (dimensionless quantities such as proportions, chances, and probabilities), and extensive quantities such as (distance, volume, and weight). But solving probability problems using these quantities exclusively is not always possible.

People have an inborn preference to use visual information. Children and adults are attracted to symmetric faces (Thornhill \& Gangested, 1999; Zaidel \& Cohen, 2005). Many art effects have symmetric structure and they most likely reflect an old approach that preceded thinking and is seen still today in animal behavior. People tend to see symmetry and they translate it to perceptual symmetry (MacBeth, Schiano \& Tversky, 1997). Maybe it has an expression in the work of the gestalt psychologists especially with regard to the holistic approach of perception (Buffart, Leeuwenberg \& Restle, 1981). 
The sensitivity to symmetry of the human visual system in general, and students' sensitivity, in particular, when solving probability problems is prevalent in this research and is related to the use of rules for estimating probabilities. It is particularly a characteristic of intermediate and low level students. Students with high grades solve most problems by calculations. The selection of rules is related to the numerical symmetry (asymmetry) of the representation of objects in the set being considered in a particular question. Students rely on visual symmetry and numerical symmetry and use a single dimension or two dimensions that are insufficient for a full description of the combinatorial relations and proportions of objects in the set being considered, or for solving the problem. They use inappropriate explanations based on extensive quantities- the relations and differences among the elements in the sets to explain their use of rules; for example they used explanations such as: "it is the same", "the numbers are close", "the numbers are far", "the number is bigger", "the numbers are the same" etc'.

The mental representation of objects in the problem is what in large part determines the set of rules that is being used to choose rules for solving the probability problem when there is a symmetric or asymmetric set of objects.

When there are small numerical differences among the different objects the intermediate and lower level students choose rules which are suitable for estimating probabilities from symmetric sets. When there are numerically large and intermediate differences among the objects they choose rules for estimating probabilities from asymmetric sets.

One possible explanation for this behavior is that the ideational knowledge of people for estimating probabilities is more suitable for visually and numerically asymmetric sets of objects, more than for numerically or visually symmetric sets of objects. The symmetry of objects in the world is most often in one visual axis (Borenstein \& Davis, 1984; Freyed \& Tversky, 1984) and less prevalent in two and three dimensions. For example, the symmetry is with relation to a visual vertical axis in the faces of people and animals, leaves and trees. Flowers have more symmetry in more axes. People have a tendency to notice minor information and especially information of the most symmetric order (Buffart, Leeuwenberg, \& Restle, 1981; Wertheimer, 1958). It is possible that symmetry has a certain value related to survival, sometimes the identification of two sided symmetry could stir the entire fear mechanism for reducing tension (Livio, 2006).

People have an inborn preference for using visual information and it has been proven that children and adults are attracted to symmetric faces (Thornhill \& Gangested, 1999; Zaidel \& Cohen, 2005). Many artifacts have symmetric structures possibly because it reflects a pre-cognitive approach that had been prevalent in human thinking thousands of generations ago (not operating under system 2), and is still prevalent in animals today. People have a tendency to notice symmetry and to translate it into visual symmetry (Freyed \& Tversky, 1984; McBeath Schiano \& Tversky, 1997; Buffart Leeuwenberg \& Restle, 1981; Goldmeier, 1972; Wertheimer, 1950). There is a sensitivity of the human visual system to symmetric shapes (Borenstein \& Krinsky, 1985; Royer, 1981) and there is evidence that people have extra sensitivity for distortion of symmetry in the vertical axis (Goldmeier effect).

The sensitivity to symmetry makes it possible for people to identify a shift from symmetry. For example, babies spend more time looking at symmetric faces of adults compared with less symmetric faces (Bornstein, Ferdinandsen \& Gross, 1981; Geddes, Jeffery \& Clark, 2002).

The findings in the current research show that students rely on the visual symmetry, numerical symmetry, and the deviation from them and are using one or two dimensions which are insufficient for a full combinatory and proportional description of the probability problem.

\subsection{Consistency When Solving Probability Problems}

Consistency in the present research is a pattern of action that characterizes students' problem solving in similar situations over a period of time.

In the pattern of action, it is meant that a student solves problems by calculations, rules, or a combination of both, or any other way for solving the problem. Consistency is the tendency to use again and again the same pattern, according to the following measures: 1) Consistency of groups of students (high grade students, intermediate grade students and low grade students): solving problems using the rules or calculations in at least $50 \%$ of the problems. 2) Personal consistency: solving problems using the rules or calculations in at least $50 \%$ or more of the problems.

Students with high grades in the written test are consistent in calculating probabilities. The explanation for the consistency in the high grade group is in accordance with the classification of Piaget and Inhelder (Piaget \& Inhelder, 1975): "in the third stage of development children have the ideas of order in a set of beads and an understanding of the mixtures from which the beads are being pulled." When they are asked to take out a pair of 
beads they can follow the structure of the mixture. The basic components and tools for estimating probabilities exist; it can be considered that the reason for the pattern of action of the children with high grades in solving probability problems is the use of calculations. They connect between what they studied in school and the problems they are given and they solve the problem by doing calculations.

Students with intermediate and low grades are consistently using estimation rules for solving probability problems when there are numerical asymmetry and symmetry of objects in the problems being considered. In the second stage of development according to Piaget and Inhelder children have not yet developed existing cognitive structures sufficient to handle the complexity of the mixture from which the beads are drawn. The explanation for consistency in the use of rules among the intermediate and low-grade students is that students use their inclination to see numerical symmetry and asymmetry in the set of objects in the problems and incorrectly match that information with the probability of drawing pairs of objects.

\subsection{The Connection between the Use of Rules and Correct Answers}

Because students with intermediate and low grades in the interviews used rules consistently and there were correct answers, I examined the correlation between the use of rules and correctness of the answers. The findings were that there was no significant statistical connection between the use of rules and the correctness of answers.

Explanation: in some of the problems the use of rules leads to correct answers since the structure of the questionnaire is such that when a correct answer is being selected it may happen at random, and not because the rule is correct.

Students with intermediate and low grades solve probability problems using extensive quantities that are not suitable for that purpose; they think in terms of system 1. On the basis of the findings of students with intermediate and low grades, I suggested a probable mechanism for probability estimations; its components will be given next.

\subsection{Elements of the Mechanism for Probability Estimations}

In the next section the elements in the mechanism for solving probabilities is presented; they are arranged not necessarily by their importance in the mechanism. The elements of the mechanism that are summarized below show the main components in problem solving and their relations.

\subsubsection{Elements of the Mechanism}

a. Seeing symmetry (the deviation from symmetry).

b. Representation of the problem. Visual representation of the problem (such as: numerically symmetric and asymmetric sets of objects). Internal representation of the problem such as: similarity, equality, proximity.

c. Extensive and intensive quantities being used by students for estimating probabilities.

d. Cognitive systems being used: system 1 and system 2 .

e. Procedural knowledge and ideational knowledge related to probability.

f. Rules for estimating probabilities (distance comparison rules, summation rules, counting rules, etc'.)

4.3.2 Explanation for Some of the Elements in the Mechanism

a. Seeing symmetry leads students to measure the deviation from symmetry and thus use inappropriate elements for solving the problems.

b. Asymmetric and symmetric representation of sets of objects lead students with low and intermediate achievements to use extensive quantities such as distance and choose rule number 2 and its variation for solving problems. Students are also using rule number 1 and its variations (extensive quantities) for solving problems with symmetric object arrangements.

c. Students tend to use the false contention that numerical distances of objects and the probability to draw objects are related and thus the concept of probability is being distorted. The estimations of probabilities are being made by using ideational knowledge and non-proper mathematical principals; for example, students think that "The greater the distance among the elements, the higher the chances of drawing particular pair." These rules are incorrect from the point of view of probability theory.

\subsubsection{The Mechanism Structure}

The proposed mechanism is used by students with intermediate and low grades for solving problems. 
The mechanism structure presents the flow of decision making processes of students solving the probability questions of drawing a pair of beads from a bag containing a mixture of colored beads, numerically symmetric or asymmetric $<$ fig $1>$. A random selection of answers or some other unknown process characterizes the decision making in approximately one third of the solutions given by the students. In these cases the mechanism proposed here does not describe the decision making processes completely.

In the present research it is seen by examining the findings that only a minority of students learned probability. Most other students used heuristics rules and random selection of answers to solve probability problems. Students who participated in probability lessons for the national examination in mathematics did not calculate probabilities, and instead used their ideational knowledge which usually is manifested by a set of rules to estimate the probabilities. In the interviews less than a quarter of the students who learned probability calculated probabilities and more than a third of students preferred to estimate probabilities using their ideational knowledge. One of characteristics for solving probability problems in the present research is the double use of calculations and probability estimation rules by students with high and intermediate grades. This is especially apparent when students are solving problems with numerical symmetry of objects and when they are asked to estimate the color of two beads drawn at random.

The present research raises several questions concerning the estimations of probabilities and problem solving of students with intermediate and lower grades: why do students not use their knowledge in probability when solving probability problems? Why do students who use calculations also use rules for solving certain problems? One of the phenomena described by researchers, which was also relevant in this research when solving problems, is the sensitivity of people to symmetry. The question that comes to mind is whether there is also sensitivity to symmetry in other areas of mathematics and what importance the sensitivity to symmetry has on learning mathematics in general and probability in particular.

Students with lower and intermediate grades who participated in probability lessons are more sensitive to symmetry and are not using their formal knowledge for solving probability problems, but rather use rules that are a part of their ideational knowledge and specific to probability when they solve questions with numerically symmetric object arrangement. They estimate that pairs of objects will be drawn with equal probability in a random drawing and in a higher probability compared to drawing from asymmetric object arrangements. The probability problem solving by students in these cases is based on the numerical distance of the objects and influenced by visual elements, the domain specific ideational knowledge of students in probability, and the procedural knowledge for estimating the drawing of objects from numerically symmetric and asymmetric sets of objects. The knowledge for estimating the drawings of objects in theses situations is expressed by the students using rules for estimating probabilities based on the numerical representation of objects in the sets as extensive quantities and, as a result, these students solve the problems in terms of elements characteristic of system 1.

Insert Figure 1 Here

\subsection{Limitation of the Research}

One limitation is the style of questions in the interviews which are designed according to the style students are required to answer on their national mathematics examination. The style was requested by the representative of the ministry of education who gave me the permit to enter schools to conduct the interviews. It creates a situation in which a student might be choosing an answer rather than working out the probabilities required. In the interviews this was compensated by asking students to explain their answers.

There is probably a large gap between the principles of probability presented to students in the class and the format in which the questions were presented to them. It is therefore likely that the mathematics activity in the probability class does not support the development of rational problem solving strategies, and in fact it supports activity which is disconnected from the structure of the problems. It seems that this situation contributes to the use of heuristics approach and support less formal problem solving.

\section{Acknowledgment}

The research includes parts of the author $\mathrm{PhD}$ thesis at Ben-Gurion University. My thesis supervisor was Professor Ron Hoz whom I would like to thank for his patient and support.

\section{References}

Borenstein, M., \& Davis, S. (1984). "Discrimination and memory for symmetry in young children". Developmental Psychology, 20, 637-649. http://dx.doi.org/10.1037/0012-1649.20.4.637

Bornstein, H., Ferdinandsen, K., \& Gross, C. (1981). Perception of symmetry in infancy. Developmental 
Psychology, 17, 82-86. http://dx.doi.org/10.1037/0012-1649.17.1.82

Bornstein, H., \& Krinsky, J. (1985). Perception of symmetry in infancy: The salience of vertical symmetry and the perception of pattern wholes. Journal of Experimental Child Psychology, 39, 1-19. http://dx.doi.org/10.1016/0022-0965(85)90026-8

Buffart, H., Leeuwenberg, E., \& Restle, F. (1981). Coding theory of visual pattern completion. Journal of Experimental Psychology: Human Perception and Performance. 7, 241-274. http://dx.doi.org/10.1037/0096-1523.7.2.241

Evans, J. (2003). In two minds: Dual process accounts of reasoning's. Trends in Cognitive Sciences, 7,459-454 . http://dx.doi.org/10.1016/j.tics.2003.08.012

Fryed, J., \& Tversky, B. (1984). Force of symmetry in form perception. American Journal of psychology, 97(1), 109-126. http://dx.doi.org/10.2307/1422551

Geddes, R., Jeffery, K., \& Clark, D. (2002). Are average and symmetric faces attractive to infants? Discrimination and looking preferences. Perception, 31(3), 315-321. http://dx.doi.org/10.1068/p3129

Goldmeier, E. (1972). Similarity in visually perceived forms. Psychological Issues, 8(1), 1-136.

Kahneman, D., \& Frederick, S. (2002). Representativeness revisited: Attribute substitution in intuitive judgment. In T. Gilovich, D. Griffin, D. Griffin, \& D. Kahneman. Heuristics and biases (pp. 379-396). Cambridge: University Press.

Leyton, M . (1992). Symmetry causality mind. Cambridge, MA: MIT Press.

Livio, M. (2006). The equation that couldn't be solved. Simon and Schuster, Inc., New York.

McBeath, M., Schiano, D., \& Tversky, B. (1997). Three dimensional Bilateral symmetry bias in judgments of figural identity and orientation. Psychological Science, 8(3), 217-233. http://dx.doi.org/10.1111/j.1467-9280.1997.tb00415.x

Piaget, J. (1964). The child's conception of number. London: Routledge \& Kegan Paul.

Piaget, J., \& Inhelder, B. (1975). The origin of the idea of chance in children. New York: W. W. Norton \& Company Inc.

Poggio, T. (1984). Vision by man and machine. Scientific American, April, 106. http://dx.doi.org/10.1038/scientificamerican0484-106

Pinker, S. (1999). How the mind works. New York: W. W. Norton \& Company Inc.

Royer, L. (1981). Detection of symmetry. Journal of Experimental Psychology: Human Perception \& Performance, 7(6), 1186-1210. http://dx.doi.org/10.1037/0096-1523.7.6.1186

Schnarch, D. (1998). Intuition and Schemata in Probabilistic Reasoning-The Evolution with Age of Probabilistic Misconceptions. Thesis Submitted for the Degree "Doctor of Philosophy", in Hebrew. Tel Aviv, Israel: Tel-Aviv University.

Schreiber, J. (2003). Cognitive difficulties of adolescents in solving probability tasks. MA Thesis, in Hebrew. Tel Aviv, Israel: Tel-Aviv University.

Thornhill, R., \& Gangestad, W. (1999). Facial attractiveness. Trends in Cognitive Sciences, 3(12), 452-460. http://dx.doi.org/10.1016/S1364-6613(99)01403-5

Tversky, A., \& Kahneman, D. (1971). Belief in the law of small numbers. Psychological Bullitin, 76, 105-110. http://dx.doi.org/10.1037/h0031322

Tversky, A., \& Kahneman D. (1974). Judgment under uncertainty: Heuristics and biases. Science, 185, 1124-1131. http://dx.doi.org/10.1126/science.185.4157.1124

Wertheimer, M. (1950). Laws of organization in perceptual forms. In W. D. Ellis (Ed.), A source book of Gestalt psychology (pp. 71-88). New York: Humanities Press.

Wertheimer, M. (1923/1958). Principles of perceptual organization. In D.C. Beardless, \& M. Wertheimer (Eds.), Readings in perception (pp. 115-135). Princeton, NJ: Van Nostrand.

Zaidel, W., \& Cohen, A. (2005). The face beauty and symmetry: Perceiving asymmetry in beautiful faces. International. J. Neuroscience, 115, 1165-1173. http://dx.doi.org/10.1080\%2F00207450590914464 
Table 1. The rate of using rules for estimating probabilities from asymmetric sets of objects in the interviews

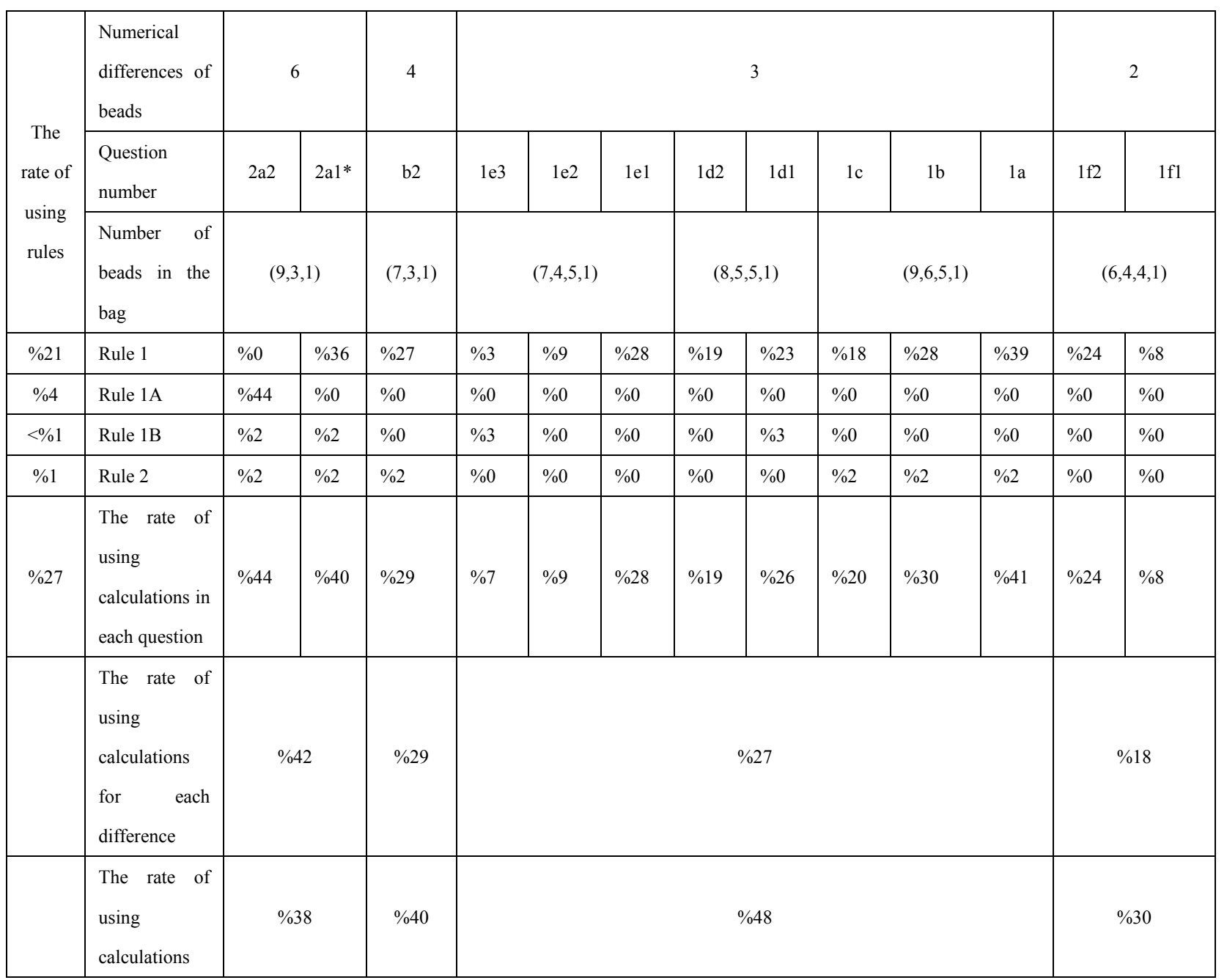

*In question 2a1 the use of rule 1 leads to a correct answer. The basic set in question 1 is composed of $(9,6,5,1)$ beads with numerical difference 3 in questions a-c, in questions d-f the drawing of beads is without returning them to the bag. In question $2 \mathrm{a} 2$ the probability estimation is with respect to objects that are not in highest number, this is probability estimation of the second kind. 
Table 2 . The rate of using rules for estimating probabilities of objects drawn from numerically symmetric sets in the interviews

\begin{tabular}{|l|l|l|l|l|l|l|}
\hline $\begin{array}{l}\text { Question } \\
\text { Number }\end{array}$ & $\begin{array}{l}\text { The rate of } \\
\text { using rules } \\
\text { for } \\
\text { estimating } \\
\text { probabilities }\end{array}$ & \multicolumn{1}{|c|}{7} & \multicolumn{2}{|c|}{6} & 5 & 4 \\
\hline $\begin{array}{l}\text { Object } \\
\text { relations } \\
\text { in the bags }\end{array}$ & $(8,9)(5,7)$ & $\begin{array}{c}(4,2)(99,100) \\
(100,99)\end{array}$ & $\begin{array}{c}(100,100)(3,4) \\
(4,3)\end{array}$ & $\begin{array}{c}(4,3)(400,400) \\
(300,300)\end{array}$ & $\begin{array}{c}(4,4)(3,4) \\
(3,4)\end{array}$ \\
\hline Rule 2 & $\% 32$ & $(\% 28)$ & $(\% 35)$ & $(\% 15)$ & $(\% 21)$ & $(\% 53)$ \\
\hline Rule 2a $>$ & $\% 17$ & $(\% 20)$ & $(\% 14)$ & $(\% 18)$ & $(\% 36)$ & $\% 0$ \\
\hline Rule $2 \mathrm{~b}<$ & $\% 3$ & $\% 0$ & $(\% 2)$ & $\% 0$ & $(\% 9)$ & $(\% 4)$ \\
\hline Rule 1c & $\% 2$ & $(\% 2)$ & $(\% 2)$ & $(\% 3)$ & $(\% 3)$ & $(\% 2)$ \\
\hline $\begin{array}{l}\text { The rate of } \\
\text { using rules } \\
\text { by } \\
\text { intermedia } \\
\text { te students }\end{array}$ & $\% 54$ & $\% 51$ & $\% 53$ & $\% 39$ & $\% 70$ & $\% 59$ \\
\hline $\begin{array}{l}\text { The rate of } \\
\text { correct } \\
\text { answers in } \\
\text { the } \\
\text { interviews }\end{array}$ & & $\% 39$ & $\% 43$ & $\% 49$ & $\% 52$ & \\
\hline
\end{tabular}


Object representation. The number of objects their type and visual perceptions as related to visual perception formal and ideational knowledge.
Solving Probability problems

1. Objects. The number of objects with different colors or a different marker e.g. A, B, C, D with numerical relations $\mathrm{a} \geq \mathrm{b} \geq \mathrm{c} \geq \mathrm{d}$.

2. Need to solve probability problem. Estimate the drawing of two objects from a set of objects at random.

3. Variables. The number of objects and the numerical relations among them.



Figure 1. Solving probability problems in a unified and uniform field 